\title{
Effect from Accessories on Pickup Aerodynamics by Computational Fluid Dynamics
}

\author{
Prachya Mukda
}

\begin{abstract}
This study aims to investigate the aerodynamic characteristic of the pickup truck. The pickup box and around body of the car has been modified and tested for drag coefficient by using low speed wind tunnel and using 3 dimensional computational fluid dynamics (CFD) technique (FLUENT). Models are tested at speed between $36-90 \mathrm{~km} / \mathrm{hr}$ in the wind tunnel and CFD, before full dimension testing at speed between $36-126 \mathrm{~km} / \mathrm{hr}$ in CFD. Six car models, which are (a) Normal, (b) Wildtrak, (c) Covered pickup box, (d) SUV, (e) Front grill and (f) Sedan and all models have the same frontal area. In this study, the main focus is to investigate the aerodynamic characteristics to reduce the coefficient of drag $\left(C_{D}\right)$ due to the wake at the rear body of the pickup car. The result shows that, almost case studies decrease drag coefficient from normal case between $5 \%-33.3 \%$ (in all average speed), except SUV case increases the drag coefficient about $10 \%$. This study confirms that reduction of internal flow in to the cab or reduce wake by the geometry at the rear body of the pickup can significantly affect the $\mathrm{C}_{\mathrm{D}}$, hence the energy economizes.
\end{abstract}

Keywords - aerodynamics, pickup, drag coefficient, computational fluid dynamics.

\section{INTRODUCTION}

$\coprod_{\mathrm{of}}^{\mathrm{N}}$ $\mathrm{N}$ Thailand, the automotive industry is targeted to be "Detroit of Asian". Now, it is the world's largest market and manufacturer of the pickup truck. In manufacturing, the truck shape is generally designed by using fundamental of aerodynamics to provide low coefficient of drag, because this can improve energy efficiency of the trucks. However, many users modified their pick-up truck with accessories such as cover the rear box or bed. This changes the pick-up truck's aerodynamic and coefficient of drag which was designed by manufacturer. Therefore, it may cause an exaggerate energy consumption. Consequently, the study of aerodynamics characteristics over the truck is beneficial to the users for improving fuel efficiency.

In the investigation of aerodynamics characteristics, wind tunnel is the most popular research tool using to figure out the aerodynamic of objects. It is also used to observe the air flow phenomena around object such as separation which is directly relate to the coefficient of drag.

Prachya Mukda, Department of Mechanical Engineering, Faculty of Industrial Technology, Phetchaburi Rajabhat University, Thailand.
In 2001, Grosche, F.R. et al. presented that object shape like buff body slightly created the separation of airflow; hence, the coefficient of drag was low because of lower differential pressure across the object. This fundamental was sometimes applied to specify the pickup geometry before such model was tested in the wind tunnel. For many times, however, because of the dissimilarity between prototypes and model in the detail, the errors in testing and experimental results are found. Therefore, in 1999, Van Dam, C.P.[2] used the larger wind tunnel, where the full size car can be tested, for aerodynamics and quantifying the coefficient of drag. Aerodynamic drag is mainly affected not only by the truck body, but also the accessories, especially which stick out from the body and is perpendicular to air flow direction. Those accessories cause the loss of energy and the reduction of air momentum along the accessories in airflow path. Although in 2006 Leuschen, J. et al. [3] presented that some of accessories can reduce car fuel consumption, around $5-7 \%$, by reduction of coefficient of drag from vortices phenomena, they did not consider the air flow inside the car which also relates to drag coefficient. Moreover, it is difficult to investigate the effect of airflow on inside the car on aerodynamic drag by using of similarity.

Recently, numerical method such as Computational Fluid Dynamics (CFD) has been employed to investigate the air flow characteristics around object and then to predict the coefficient of drag of car. At the beginning, domains of 2D geometry were simulated with low Reynolds number to capture the separation of airflow at the rear of object. Such model was comparable to similarly model tested in wind tunnel. However, in the full dimension test, it is different between the results from simulation and experiment, because, the effect of the third dimension of the geometry is not considered. Thus, Horinouchi, N. [4] and Muyl, F. et al.[5] improved CFD model of car by using Navier Stoke equation of laminar flow of air and applying the domain to 3D geometry to investigate the airflow characteristics over car accessories. Moreover, they found that drag force of car shape is significantly decreased with high slop of object front. Further study, Prasad, A. et al [6], confirmed that drag force can be reduced by installation of front skirt, and they mentioned that $40 \%$ of coefficient of drag was accounted by the outside force which was mostly generated by turbulent flow in the rear of the car.

Muyl, F. et al.[5] successfully carried out the simulation of characteristics of airflow around the car by using the numerical model with car geometry divided into parts of front, middle, and rear. The part of rear was modified in major angle of car shape, 
being back-light angle, boat-tail angle and ramp angle. He found that boat-tail angle significantly more induces wake flow generation, corresponding to the drag coefficient. Moreover, many results from the above previous studies indicates that drag coefficient of pickup truck shape is higher than which of another car without bed, because of wake flow more rapidly occurring over the tray as shown in Fig 1 [7].

Drag forces of pickup can be divided as four parts in Fig 2. Which pressure drag occur highest to 77 percentage, internal flow loss 10 percentage, under floor roughness 10 percentage and friction loss occurs around body car about 3 percentage [8].

This study carries out an investigation of airflow characteristics over the pickup truck focused on the pickup box or bed for improving the body aerodynamics. The pickup truck models are tested at low speed in the wind tunnel, before the full dimensions are tested at high speed in CFD simulation. Six car models, which are (a) Normal, (b) Wildtrak, (c) Covered pickup box, (d) SUV, (e) Front grill and (f) Sedan are investigated. Moreover, the aerodynamic characteristics corresponding to the coefficient of drag $\left(\mathrm{C}_{\mathrm{D}}\right)$ due to the wake flow at the rear part is discussed.

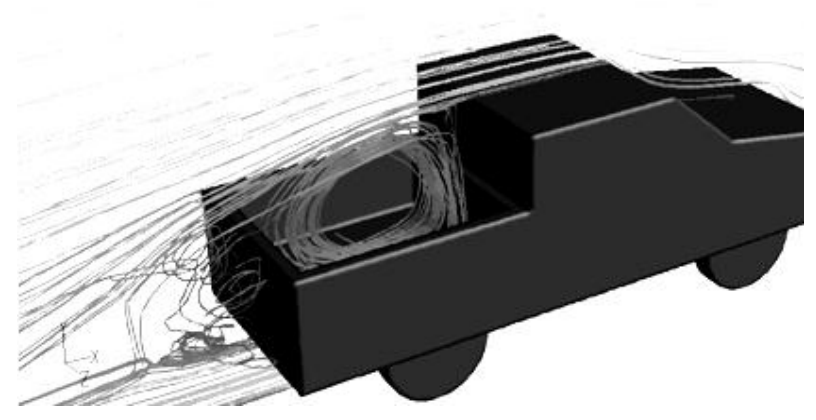

Fig. 1 Wake flow over the bed of pick-up track [7]

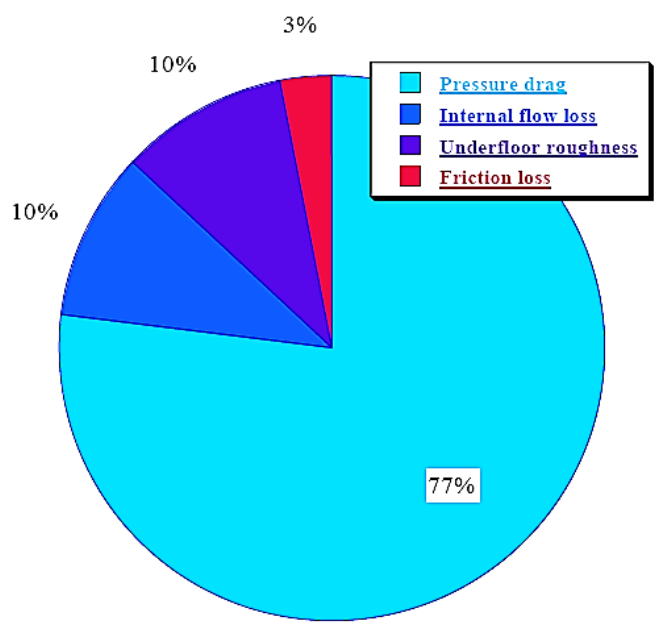

Fig. 2 Proportion of drag on the car [8]

\section{MethodOLOGY}

\section{A. Evaluation of Turbulent Model}

In this study, the commercial CFD code (FLUENT) is used as the tool to simulate the aerodynamic characteristics of objects. In the simulation, the domain is assumed to be 3D geometry with 728,000 triangle grid or tetrahedron mesh element. At the beginning, various turbulent models for specifying the turbulent flow were evaluated by comparison with previous experimental. It shows that simulation of coefficient of drag of validation by using k-e model gives fairly close results to experimental results [9]. Therefore, this turbulent model is employed in this paper.

\section{B. Testing Model in the Wind Tunnel}

Low speed wind tunnel used in this study is shown in Fig.4. Air from inlet section is induced through the test section by the fan with the motor sized of $5.5 \mathrm{hp}$. The rotational speed of fan can be regulated by the inventor to obtain air velocity of around $10-25 \mathrm{~m} / \mathrm{s}(36-90 \mathrm{~km} / \mathrm{hr})$. Velocity and pressure of airflow in the test section is measured by the pitot tube and the barometer. Fig. 4, on right hand side, shows the measurement of drag force of the sphere with $0.1 \mathrm{~m}$ in diameter by using load cell and manometer. Thus, the drag coefficient of the shape can be found from Equation (1) as

$$
C_{D}=\frac{F_{D}}{\frac{1}{2} \rho V^{2} A}
$$

where $F_{D}$ is the total drag force acting on an object, $\rho$ is the density of air and $A$ is the frontal area of the pickup.

\section{Comparison between Experiment and Simulation (CFD)}

\section{Pick-up truck model}

Principle of similarity was used to specify dimensions of the pickup truck models, which would be tested in the wind tunnel and calculated by the CFD method. The ratio of the model to the actual size of pick-up truck is 1:20. The model was made of metal which the frontal area (and shape) was fixed at $0.0067144 \mathrm{~m}^{2}$. Air velocities ranged between $36-90 \mathrm{~km} / \mathrm{hr}$ were used in both the simulation and the experiment.

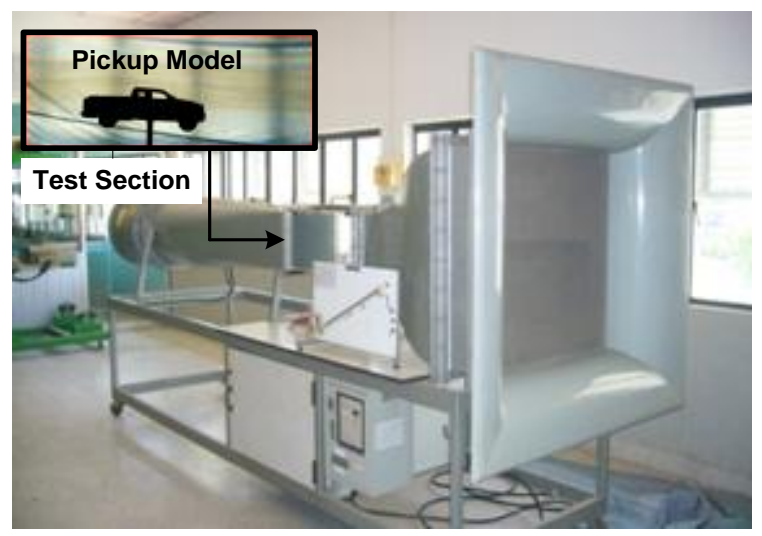

Fig. 3 Wind tunnel for the model car testing [3] 
D. Prediction for Coefficient of Drag of Pickup Truck with Full Scale Test

For full scale test, the 6 cases of pickup trucks detailed above section are calculated by CFD method to estimate the coefficient of drag, where the frontal area of the pickup truck was fixed on $2.68576 \mathrm{~m}^{2}$. Moreover, influence of airflow inside the driver room or cab on the coefficient of drag is also investigated. Air speed ranged $36-126 \mathrm{~km} / \mathrm{hr}$, corresponding to Reynolds number between $2.6 \times 10^{4}-2.5 \times 10^{5}$, is used with the same CFD setup as in the previous simulations. There are 728,000 triangle or tetrahedron mesh elements with inlet and outlet boundary condition defined as velocity inlet and atmospheric pressure outlet respectively. Which the condition and domain are shown in Table 1 and Fig. 4, respectively.

\begin{tabular}{ll}
\multicolumn{2}{c}{ TABLE I } \\
\multicolumn{1}{c}{ BAMBNDARY CONDITION OF CFD } \\
\hline inlet boundary condition & velocity inlet \\
outlet boundary condition & pressure outlet \\
grid triangle & 728,000 cell \\
\hline FLUENT 3D \\
\hline solve & segregated Solver \\
linearization & implicit method \\
turbulent model & standard \\
& k-epsilon \\
near-wall treatment method & standard \\
velocity testing & wall function \\
\hline
\end{tabular}

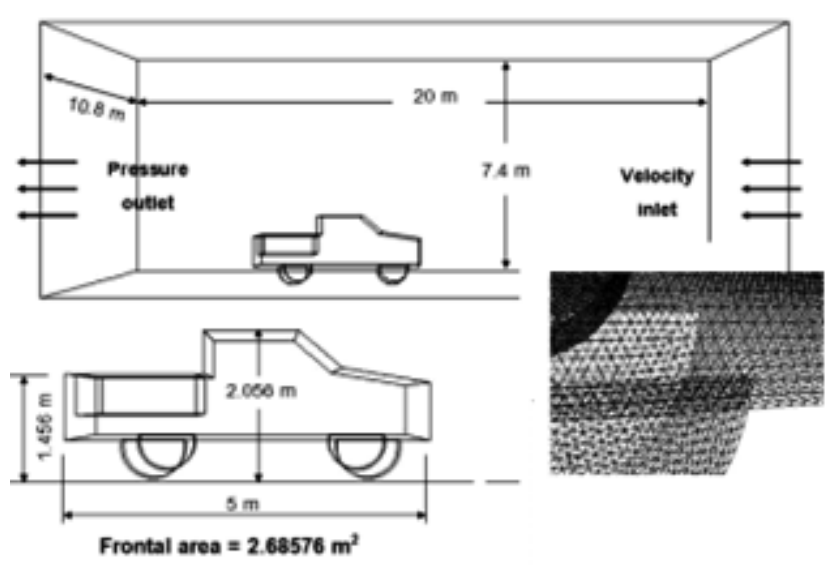

Fig. 4 Domain of the simulation setup with the real dimension pickup truck

\section{Result AND Discussion}

\section{A. Validation Result}

In the simulation, according to the validation in the previous section, the k- $\varepsilon$ turbulent model is used. Value of coefficient of drag of the spherical from the simulation, the experiment, and the theoretical results are compared at different Reynolds number ranged from $2.2 \times 10^{4}-3.0 \times 10^{5}$, as shown in Fig. 5 . It is found that the coefficients of drag from those methods are quite similar. The coefficient from the experiment is higher than that from the simulation by $7 \%$ averagely. The drag coefficient decreases when the Reynolds number increases, because the viscous friction force acting on spherical surface decreases.

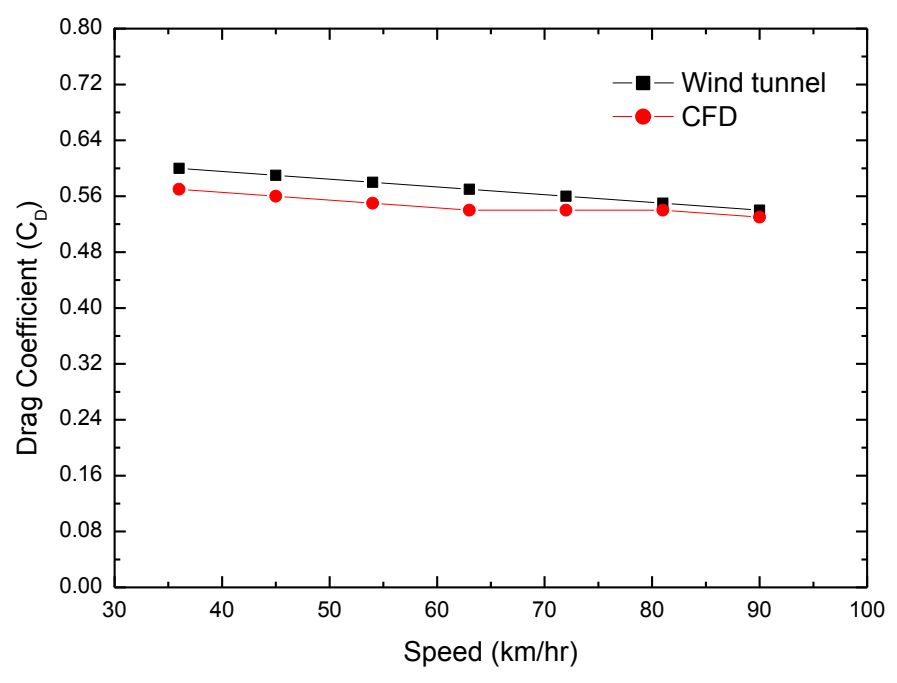

Fig. 5 Coefficients of drag from validation of normal case model

\section{B. Comparison and discussion of the pickup 6 cases}

The pickup trucks whose geometry provides the low drag force. Therefore, in automotive design, the designer generally strives to achieve the low coefficient of drag by reducing of pressure in frontal part of truck and increasing of pressure in the rear part. In this study, the concept of pressure or force difference over the truck body can be applied to describe the aerodynamics drag of all cases of pickup trucks by using Equation (2). The coefficients of drag and path line of air flow from CFD simulation are shown in Fig. 6 and Fig. 7 respectively. We found that the SUV case performs with the highest coefficient of drag, and the Sedan case performs with the lowest coefficient of drag.

$$
F_{D}=\int_{\text {front }} p \cos \theta d A-\int_{\text {back }} p \cos \theta d A
$$

where $F_{D}$ is total drag force acting on the pickup $(\mathrm{N}), p$ is air pressure (pascal) and $A$ is frontal area of pickup $\left(\mathrm{m}^{2}\right)$ 


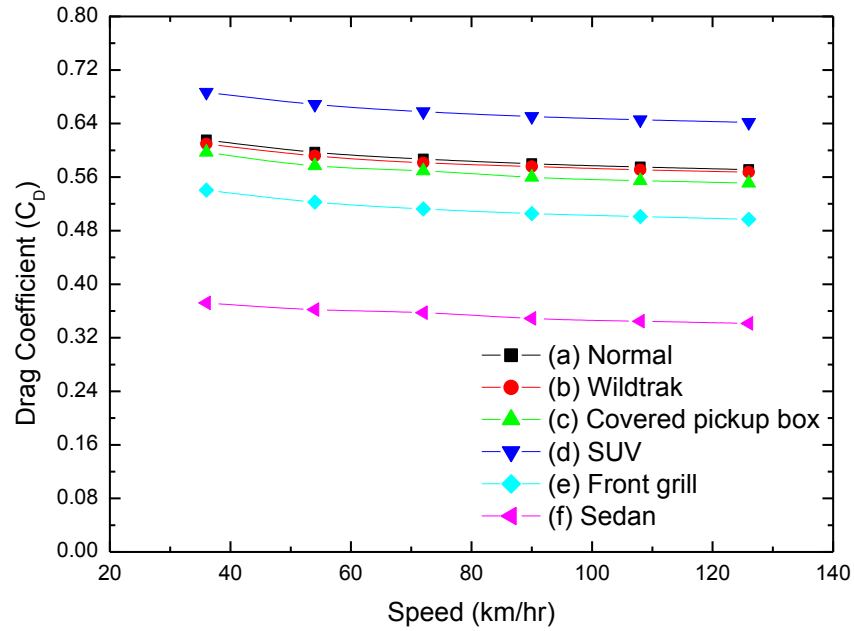

Fig. 6 Drag coefficient on increasing speed of pickup

\section{Pressure pathline of airflow over the pickup}

\section{Case (a): Normal}

Normal case is used to compare to other study cases which is the commercial pickup accessories. From the results, the pressure pathline of airflow can be investigated to discuss for drag coefficient. In Fig. 7 (a) is observed that, when the air pressure flow impact to the front area, causes separation and recirculation air on pickup box, it's called "wake", which in this case have large size because high different of pressure between front to rear of pickup, which can be discussed from Equation (2). When the wake impacts to the inside of the box causing the total drag force of pickup is high. Therefore, the generally normal case has high drag coefficient value.

\section{Case (b): Wildtrak}

In this case study, the commercial accessories is stuck at top and rear of the pickup cabin. The result shows that, this case have nearby drag coefficient to normal case, due to the wake flow at the rear box change lightly.

\section{Case (c): Covered pickup box}

In the covered pickup box case, the phenomena different from two first cases is the wake size at rear box less than, because the covered pickup box can reduce difference of pressure between the front and rear of pickup. Which accord with the total drag coefficient as in the Fig. 6.

\section{Case (d): SUV}

This case obtains highest total drag force due to the SUV case is square shape accessories causing highest difference pressure. It can observe from the wake is largest more than every cases. Therefore, this case increases of the highest drag coefficient more than every case as in Fig. 6.

\section{Case (e): Front grill}

This case strict grill at the front of pickup, which decrease drag coefficient due to a certain angle of the slant, a vortex breakdown phenomenon appears causing the sudden pressure drop acting on the front pickup. Therefore the pressure drag coefficient is less than normal case.

\section{Case (f): Sedan}

In this case, pressure pathline of airflow seems smooth than others, resulting from smoother shapes of rear part. In addition, separation and wake circulation of pickup is also minimally due to the pressure between front and rear of pickup is balance; so that, this case gives the lowest coefficient of drag.

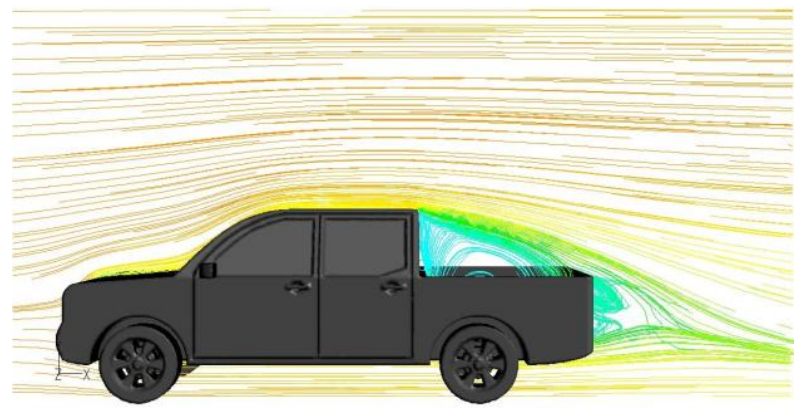

(a) Normal

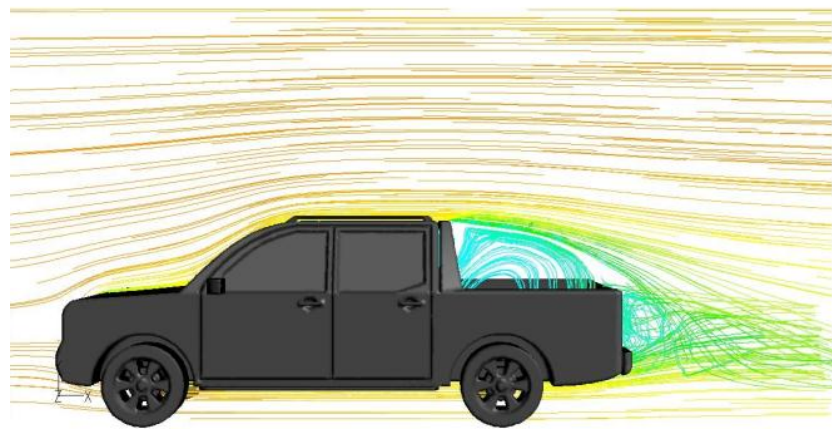

(b) Wildtrak

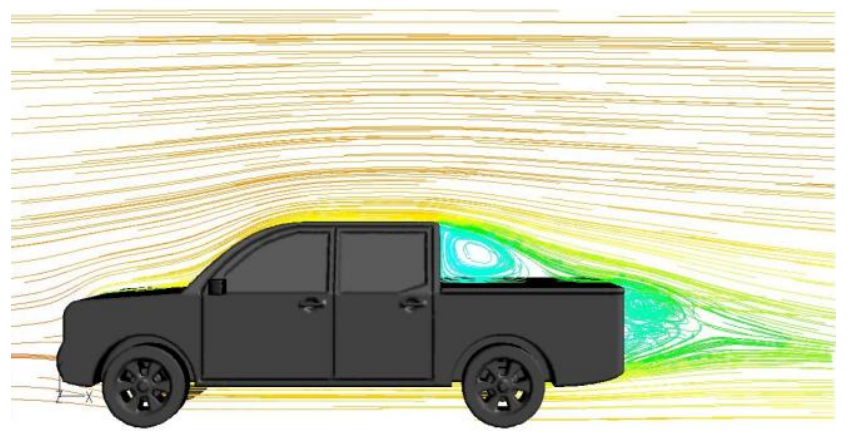

(c) Covered pickup box

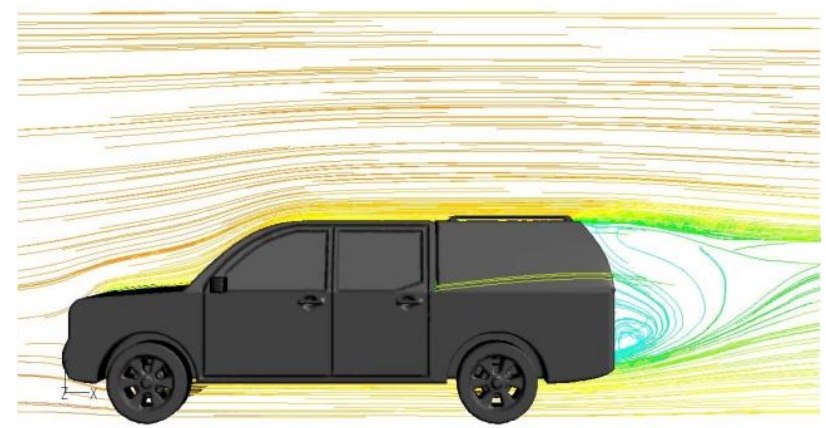

(d) SUV 


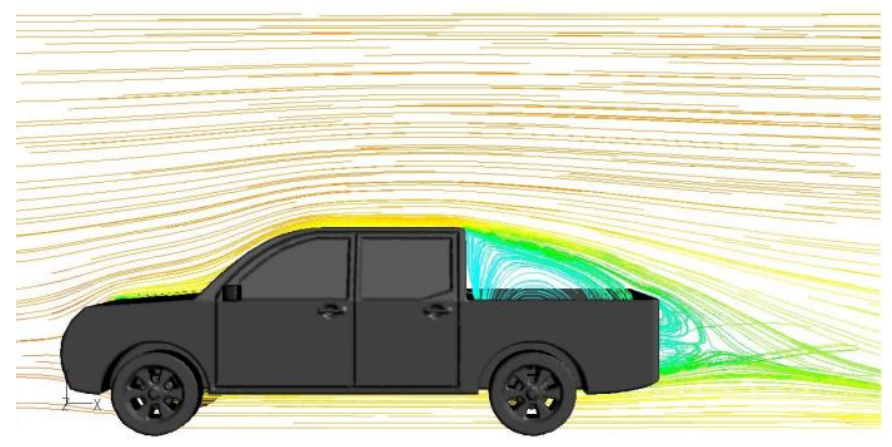

(e) Front grill

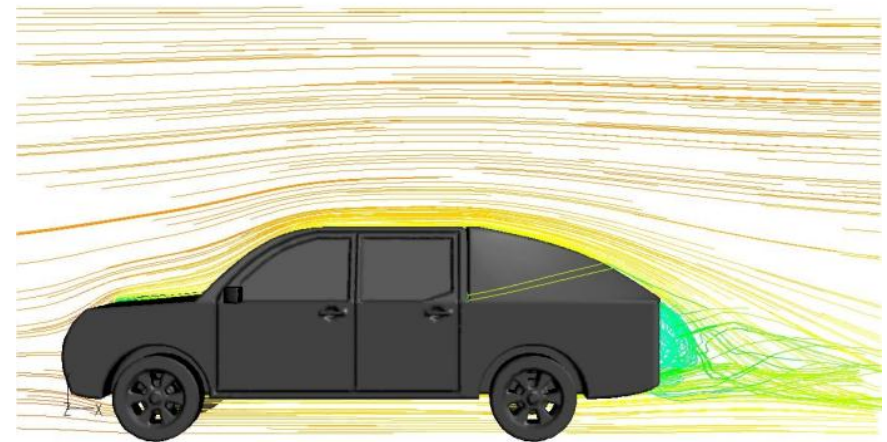

(f) Sedan

Fig. 7 Pressure pathline of 6 study cases

\section{CONCLUDING REMARKS}

This study carries out an investigation of airflow characteristics over the pickup. The pickup models are validated in wind tunnel and CFD simulation to investigate the accuracy, before using CFD modeling test the full dimension 6 cases at high speed on CFD simulation.

The validation is difference results less than $7 \%$. The drag coefficient value of sedan case decrease from normal case lowest about 0.3 , opposite the SUV case increase about 0.07 .

The results show that, the drag coefficient values $\left(C_{D}\right)$ are depended by the different between of the front pressure to rear pressure of pickup which correspond to Equation (2). Therefore, the general idea of how the drag coefficient reduction can save the fuel consumption.

\section{ACKNOWLEDGMENT}

This research is financially supported by the National Research Council of Thailand (NRCT) through Phetchaburi Rajabhat University, Thailand, 2015.

\section{REFERENCES}

[1] F.R. Grosche, G.E.A and Meier, "Research at DLR Gottingen on bluff body aerodynamics drag reduction by wake ventilation and active flow control", Journal of Wind Engineering and Industrial Aerodynamics, vol. 89, 2001, pp. 1201-1218. https://doi.org/10.1016/S0167-6105(01)00161-1

[2] C.P. Van Dam, "Recent experience with different methods of drag prediction", Progress in Aerospace Sciences, vol. 35, 1999, pp. 751-798. https://doi.org/10.1016/S0376-0421(99)00009-3
[3] J. Leuschen and K.R. Cooper, "Full-Scale Wind Tunnel Tests of Production and Prototype, Second-Generation Aerodynamic Drag-Reducing Devices for Tractor-Trailers", SAE International, 2006. https://doi.org/10.4271/2006-01-3456

[4] N. Horinouchi, "Numerical Investigation of Vehicle Aerodynamics with Overlaid Grid System", SAE 950628, 1995.

[5] F. Muyl, L. Dumsa, and V. Herbert, "Hybrid method for aerodynamic shape optimization in automotive industry", Computers \& Fluids, vol. 33, 2004, pp. 849-858. https://doi.org/10.1016/j.compfluid.2003.06.007

[6] A. Prasad and C.H.K. Williamson, "A method for the reduction of bluff bodydrag", Journal of Wind Engineering and Industrial Aerodynamics, vol. 69-71, 1997, pp. 155-167. https://doi.org/10.1016/S0167-6105(97)00151-7

[7] P. Mukda and P. Sooksanan, "Aerodynamics Analysis of Truck Front Accessories by Computational Fluid Dynamics", Journal of Science and Technology, Maha Sarakham University. vol. 34 (3), 2015 pp. 42-51.

[8] W.H. Hucho, "Aerodynamics of road vehicles," Journal of wind Engineering and Industrial Aerodynamics, vol. 94, 2006, pp. 334-361.

[9] P. Mukda A. Saysoy, "Fuel Consumption on Aerodynamics Characteristics of Pickup Accessories", Journal of Science and Technology, Ubon Ratchathani University. vol. 13(4), 2011, pp. 255262. 